\title{
Silica-Supported Phosphorus-Containing Catalysts Efficiently Promoted Synthesis of 1,8-Dioxo-octahydro- xanthenes under Solvent-Free Conditions
}

\author{
ALIREZA HASANINEJAD $^{* a}$, MARJAN DADAR $^{\mathrm{a}}$ and ABDOLKARIM ZARE ${ }^{\mathrm{b}}$ \\ ${ }^{\mathrm{a}}$ Department of Chemistry, Faculty of Sciences, Persian Gulf University, \\ Bushehr 75169, Iran \\ ${ }^{\mathrm{b}}$ Department of Chemistry, Payame Noor University, PO BOX 19395-4697, Tehran, Iran \\ ahassaninejad@yahoo.com
}

Received 5 May 2012 / Accepted 28 May 2012

\begin{abstract}
Synthesis of 1,8-dioxo-octahydroxanthene derivatives from dimedone and aldehydes using silica-supported phosphorus pentoxide $\left(\mathrm{P}_{2} \mathrm{O}_{5} / \mathrm{SiO}_{2}\right)$ or Silicaphosphine ( $\mathrm{Silphos,}\left[\mathrm{PCl}_{3-\mathrm{n}}\left(\mathrm{SiO}_{2}\right)_{\mathrm{n}}\right]$ ) as efficient and heterogeneous catalysts is described in this paper. The reactions were completed in relatively short reaction times under solvent-free conditions at $80{ }^{\circ} \mathrm{C}$ and the products were obtained in good to excellent yields.
\end{abstract}

Keywords: $\mathrm{P}_{2} \mathrm{O}_{5} / \mathrm{SiO}_{2}$, Silphos, 1,8-Dioxo-octahydroxanthene, Dimedone, Aldehyde, Solvent-free

\section{Introduction}

Recently, the use of catalysts and reagents supported on solid supports especially those based on silica and also solvent-free conditions have been extensively developed because such reagents not only cause to simplify the purification processes but also help to prevent the releasing of toxic reaction residues into the environment ${ }^{1-3}$. Moreover, due to moisture sensitivity of some reagents, application of them in organic reactions is difficult. This problem can be solved by their immobilization onto solid supports ${ }^{1-3}$. Silica-supported phosphorus-containing catalysts are one class of the most interesting solid-supported catalysts and are inexpensive, commercially available and environmentally benign which has been used in various organic transformations ${ }^{3,4}$.

Xanthene derivatives are of importance as they have been used as dyes, fluorescent materials and laser technologies due to their useful spectroscopic properties ${ }^{5}$. These compounds have also received significant attention due to their useful biological and pharmaceutical properties such as antiviral ${ }^{6}$, antibacterial ${ }^{7}$ and antinociceptive activities ${ }^{8}$ as well as their efficiency in photodynamic therapy ${ }^{9}$ and anti-inflammatory activities ${ }^{10}$. Some procedures have been reported for the synthesis of 1,8-dioxo-octahydroxanthene derivatives employing dimedone and aromatic aldehydes in the presence of acidic catalysts such as 
$\mathrm{SbCl}_{3} / \mathrm{SiO}_{2}{ }^{11}, \mathrm{HClO}_{4} / \mathrm{SiO}_{2}{ }^{12}$, silica chloride ${ }^{13}, \mathrm{FeCl}_{3} / \mathrm{SiO}_{2}{ }^{14}$, silica sulfuric acid ${ }^{15}$, Dowex$50 \mathrm{w}^{16}$, Amberlyst- $15^{17}$, [bmim] [TFA $]^{18}$ and $\mathrm{TMSCl}^{19}$. However, the reported methods suffer from drawbacks such as long reaction times, moderate yields, the use of toxic reagents, formation of by-products and no agreement with the green chemistry protocols.

In continuation of our interest on synthesis of heterocyclic compounds ${ }^{20}$, we report here a new, green and highly efficient method for the synthesis of 1,8-dioxooctahydroxanthenes via the condensation of dimedone with aldehydes using $\mathrm{P}_{2} \mathrm{O}_{5} / \mathrm{SiO}_{2}$ or silphos as non-toxic, readily producible and heterogeneous catalysts under solventfree conditions at $80{ }^{\circ} \mathrm{C}$ (Scheme 1). Interestingly, our method has none of the abovementioned disadvantages at all.

\section{Experimental}

All chemicals were purchased from Merck or Fluka Chemical Companies. All compounds are known and their structures were identified by comparing their melting points and ${ }^{1} \mathrm{H}$ and ${ }^{13} \mathrm{C}$ NMR data with those reported in the literature. The ${ }^{1} \mathrm{H}$ NMR $(500 \mathrm{MHz})$ and ${ }^{13} \mathrm{C}$ NMR $(125 \mathrm{MHz})$ were run on a Bruker Avance DPX-250, FT-NMR spectrometer ( $\delta$ in ppm). Melting points were recorded on a Büchi B-545 apparatus in open capillary tubes.

Preparation of $\mathrm{P}_{2} \mathrm{O}_{5} / \mathrm{SiO}_{2}(30 \% \mathrm{w} / \mathrm{w})$

A mixture of $\mathrm{SiO}_{2}$ [silica gel 60, 0.063-0.200 mm (70-230 mesh ASTM), $3.5 \mathrm{~g}$ ] and $\mathrm{P}_{2} \mathrm{O}_{5}$ $(1.5 \mathrm{~g})$ was ground vigorously to give the catalyst, $\mathrm{P}_{2} \mathrm{O}_{5} / \mathrm{SiO}_{2}$, as a white powder $(5.0 \mathrm{~g})^{3}$. This catalyst can be kept without any change for months in a capped bottle.

\section{Preparation of silphos}

Under an argon atmosphere, to a flask containing dried silica gel (type 60, 15-40 $\mu \mathrm{m}$ ) $(18.0 \mathrm{~g}, 300 \mathrm{mmol})$ was added $\mathrm{PCl}_{3}(13.8 \mathrm{~g}, 100 \mathrm{mmol})$ at room temperature and stirred slowly with a mechanical stirrer for $30 \mathrm{~min}$. The mixture was then heated to $60{ }^{\circ} \mathrm{C}$ while it was stirring ( 400 cycle/min) under pressure of argon for $3 \mathrm{~h}$ to remove all $\mathrm{HCl}$. The reaction mixture was washed with $50 \mathrm{~mL}$ of dry $\mathrm{CH}_{2} \mathrm{Cl}_{2}$ and dried under vacuum. Silphos was obtained as a white solid (21.6-22.3 g), which was stored in a capped bottle ${ }^{4}$. The catalyst can be kept without any change for months.

General procedure for the preparation of 1,8-dioxo-octahydroxanthenes using $\mathrm{P}_{2} \mathrm{O}_{5} / \mathrm{SiO}_{2}$ or silphos

A mixture of dimedone $(2 \mathrm{mmol})$, aldehyde $(1 \mathrm{mmol})$ and $\mathrm{P}_{2} \mathrm{O}_{5} / \mathrm{SiO}_{2}(0.12 \mathrm{~g})$ or silphos $(1.0 \mathrm{~g})$, in a round-bottomed flask connected to a reflux condenser, was heated and stirred in an oil-bath $\left(80^{\circ} \mathrm{C}\right)$. After completion the reaction as monitored by TLC, EtOAc $(10 \mathrm{~mL})$ was added to the reaction mixture, stirred for $2 \mathrm{~min}$ and filtered. The solvent of the filtrate was evaporated and the resulting solid (crude product) was recrystallized from EtOH (97\%) to afford the pure product.

\section{Selected spectral data of the products}

\section{3,3,6,6-Tetramethyl-9(phenyl)-1,8-dioxo-octahydroxanthene (Table 3, Entry 1)}

${ }^{1} \mathrm{HNMR}\left(\mathrm{CDCl}_{3}, 500 \mathrm{MHz}\right): \delta 0.90(\mathrm{~s}, 6 \mathrm{H}), 1.04(\mathrm{~s}, 6 \mathrm{H}), 2.09(\mathrm{~d}, J=16.1 \mathrm{~Hz}, 2 \mathrm{H}), 2.27$ $(\mathrm{d}, J=16.2 \mathrm{~Hz}, 2 \mathrm{H}), 2.53(\mathrm{~d}, J=17.1 \mathrm{~Hz}, 2 \mathrm{H}), 2.58(\mathrm{~d}, J=17.7 \mathrm{~Hz}, 2 \mathrm{H}), 4.53(\mathrm{~s}, 1 \mathrm{H}), 7.10$ $(\mathrm{t}, J=7.0 \mathrm{~Hz}, 1 \mathrm{H}), 7.18(\mathrm{~d}, J=7.0 \mathrm{~Hz}, 2 \mathrm{H}), 7.21(\mathrm{t}, J=7.20 \mathrm{~Hz}, 2 \mathrm{H}) ;{ }^{13} \mathrm{C} \mathrm{NMR}\left(\mathrm{CDCl}_{3}\right.$, $125 \mathrm{MHz}): \delta 27.7,29.6,32.3,32.6,41.3,51.2,116.1,126.8,128.4,128.8,144.5,162.7,196.8$. 
3,3,6,6-Tetramethyl-9(4-chloro-Phenyl)-1,8-dioxo-octahydroxanthene (Table 3, Entry 2)

${ }^{1} \mathrm{H}$ NMR $\left(\mathrm{CDCl}_{3}, 500 \mathrm{MHz}\right): \delta 0.90(\mathrm{~s}, 6 \mathrm{H}), 1.04(\mathrm{~s}, 6 \mathrm{H}), 2.09(\mathrm{~d}, J=16.1 \mathrm{~Hz}, 2 \mathrm{H}), 2.27$ $(\mathrm{d}, J=16.1 \mathrm{~Hz}, 2 \mathrm{H}), 2.52(\mathrm{~d}, 2 \mathrm{H}), 2.57(\mathrm{~d}, J=17.6 \mathrm{~Hz}, 2 \mathrm{H}), 4.51(\mathrm{~s}, 1 \mathrm{H}), 7.19(\mathrm{~d}, J=8.3$ $\mathrm{Hz}, 2 \mathrm{H}), 7.29(\mathrm{~d}, J=8.2 \mathrm{~Hz}, 2 \mathrm{H}) ;{ }^{13} \mathrm{C} \mathrm{NMR}\left(\mathrm{CDCl}_{3}, 125 \mathrm{MHz}\right): \delta 27.7,29.7,31.8,32.6$, 41.3, 51.1, 115.6, 128.6, 130.2, 132.4, 143.1, 162.8, 196.7.

\section{3,3,6,6-Tetramethyl-9(3-nitro-Phenyl)-1,8-dioxo-octahydroxanthene (Table3, Entry 6)}

${ }^{1} \mathrm{H} \mathrm{NMR}\left(\mathrm{CDCl}_{3}, 500 \mathrm{MHz}\right): \delta 0.91(\mathrm{~s}, 6 \mathrm{H}), 1.04(\mathrm{~s}, 6 \mathrm{H}), 2.11(\mathrm{~d}, J=16.1 \mathrm{~Hz}, 2 \mathrm{H}), 2.29$ $(\mathrm{d}, J=16.1 \mathrm{~Hz}, 2 \mathrm{H}), 2.57(\mathrm{~d}, J=17.9 \mathrm{~Hz}, 2 \mathrm{H}), 2.60(\mathrm{~d}, J=17.9 \mathrm{~Hz}, 2 \mathrm{H}), 4.65(\mathrm{~s}, 1 \mathrm{H}), 7.56$ $(\mathrm{t}, J=7.7 \mathrm{~Hz}, 1 \mathrm{H}), 7.66(\mathrm{~d}, J=7.6 \mathrm{~Hz}, 1 \mathrm{H}), 8.00(\mathrm{~d}, 2 \mathrm{H}) ;{ }^{13} \mathrm{C} \mathrm{NMR}\left(\mathrm{CDCl}_{3}, 125 \mathrm{MHz}\right): \delta$ 27.2, 29.6, 32.0, 32.7, 50.9, 56.6, 60.7, 106.3, 115.1, 136.8, 140.7, 153.2, 163.9, 197.0.

3,3,6,6-Tetramethyl-9(3,4-dimethoxy-Phenyl)-1,8-dioxo-octahydroxanthene (Table 3, Entry 17)

${ }^{1} \mathrm{H}$ NMR $\left(\mathrm{CDCl}_{3}, 500 \mathrm{MHz}\right): \delta 0.92(\mathrm{~s}, 6 \mathrm{H}), 1.04(\mathrm{~s}, 6 \mathrm{H}), 2.10(\mathrm{~d}, J=16.1 \mathrm{~Hz}, 2 \mathrm{H}), 2.27$ $(\mathrm{d}, J=16.1 \mathrm{~Hz}, 2 \mathrm{H}), 2.51(\mathrm{~d}, J=17.6 \mathrm{~Hz}, 2 \mathrm{H}), 2.57(\mathrm{~d}, J=17.4 \mathrm{~Hz}, 2 \mathrm{H}), 3.68(\mathrm{~s}, 6 \mathrm{H}), 4.48$ $(\mathrm{s}, 1 \mathrm{H}), 6.67(\mathrm{dd}, J=8.3,1.9 \mathrm{~Hz}, 1 \mathrm{H}), 6.72(\mathrm{~d}, J=1.9 \mathrm{~Hz}, 1 \mathrm{H}), 6.80(\mathrm{~d}, J=8.31 \mathrm{~Hz}, 1 \mathrm{H})$; ${ }^{13} \mathrm{C} \mathrm{NMR}\left(\mathrm{CDCl}_{3}, 125 \mathrm{MHz}\right): \delta 27.2,29.6,31.4,32.7,50.9,56.3,56.3,112.2,112.9,115.4$, 120.9, 137.7, 148.1, 148.9, 163.6, 196.9.

\section{3,3,6,6-Tetramethyl-9(5-bromo-2-hydroxy-Phenyl)-1,8-dioxo-octahydroxanthene}

(Table 3, Entry 19)

${ }^{1} \mathrm{H} \mathrm{NMR}\left(\mathrm{CDCl}_{3}, 500 \mathrm{MHz}\right): \delta 0.90(\mathrm{~s}, 6 \mathrm{H}), 0.97(\mathrm{~s}, 3 \mathrm{H}), 1.05(\mathrm{~s}, 3 \mathrm{H}), 2.04(\mathrm{~d}, J=15.8 \mathrm{~Hz}$, $2 \mathrm{H}), 2.25(\mathrm{~d}, J=15.9 \mathrm{~Hz}, 2 \mathrm{H}), 2.34(\mathrm{~d}, J=17.3 \mathrm{~Hz}, 2 \mathrm{H}), 2.55(\mathrm{~d}, J=17.4 \mathrm{~Hz}, 2 \mathrm{H}), 5.04$ $(\mathrm{s}, 1 \mathrm{H}), 6.95(\mathrm{~d}, J=8.64 \mathrm{~Hz}, 1 \mathrm{H}), 7.04(\mathrm{~s}, 1 \mathrm{H}), 7.28(\mathrm{~d}, J=8.60 \mathrm{~Hz}, 1 \mathrm{H}), 10.59(\mathrm{~s}, 1 \mathrm{H}) ;{ }^{13} \mathrm{C}$ NMR $\left(\mathrm{CDCl}_{3}, 125 \mathrm{MHz}\right): \delta 26.9,28.4,30.0,32.5,51.2,100.4,111.3,116.3,129.1,130.6$, $131.4,149.8,165.3,196.5$.

\section{3,3,6,6-Tetramethyl-9(2-phenyl-ethylene)-1,8-dioxo-octahydroxanthene (Table 3,} Entry 20)

${ }^{1} \mathrm{H}$ NMR $\left(\mathrm{CDCl}_{3}, 500 \mathrm{MHz}\right): \delta 1.04(\mathrm{~s}, 6 \mathrm{H}), 1.06(\mathrm{~s}, 6 \mathrm{H}), 2.25(\mathrm{~d}, J=16.04 \mathrm{~Hz}, 2 \mathrm{H}), 2.31$ $(\mathrm{d}, J=16.02 \mathrm{~Hz}, 2 \mathrm{H}), 2.52(\mathrm{~s}, 4 \mathrm{H}), 4.15(\mathrm{~d}, J=5.29 \mathrm{~Hz}, 1 \mathrm{H}), 6.19(\mathrm{~d}, J=16.05 \mathrm{~Hz}, 1 \mathrm{H})$, $6.21(\mathrm{dd}, 1 \mathrm{H}), 7.19(\mathrm{~m}, 1 \mathrm{H}), 7.27(\mathrm{~d}, J=3.7 \mathrm{~Hz}, 4 \mathrm{H}) ;{ }^{13} \mathrm{C} \mathrm{NMR}\left(\mathrm{CDCl}_{3}, 125 \mathrm{MHz}\right): \delta 27.7$, 28.3, 29.4, 32.8, 50.9, 114.1, 126.8, 128.2, 129.4, 130.4, 132.2, 137.5, 164.4, 197.1.

\section{Results and Discussion}

Initially, we studied effect of amount of the catalysts on the reaction of dimedone ( $2 \mathrm{mmol})$ with benzaldehyde $(1 \mathrm{mmol})$ under solvent-free conditions at $80{ }^{\circ} \mathrm{C}$ (Scheme1). The results are summarized in Table 1. As it can be seen in Table 1, the best amounts of $\mathrm{P}_{2} \mathrm{O}_{5} / \mathrm{SiO}_{2}$ and silphos were 0.12 and $1.0 \mathrm{~g}$, respectively. The reaction was also examined in the presence of $\mathrm{P}_{2} \mathrm{O}_{5}, \mathrm{PCl}_{3}$ and $\mathrm{SiO}_{2}$ separately (Table 1, entries 10-12); however, the product was obtained in moderate yields in these conditions.

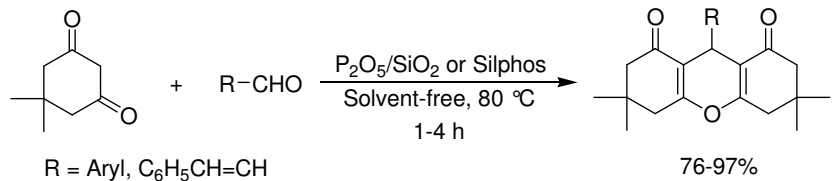

Scheme 1 
Table 1. The condensation of dimedone $(2 \mathrm{mmol})$ with benzaldehyde $(1 \mathrm{mmol})$ using different amounts of the catalysts under solvent-free conditions at $80{ }^{\circ} \mathrm{C}$

\begin{tabular}{ccccc}
\hline Entry & Catalyst & Amount, $\mathrm{g}$ & Time, $\mathrm{h}$ & Yield $^{\mathrm{a}}, \%$ \\
\hline 1 & - & - & 8 & - \\
2 & $\mathrm{P}_{2} \mathrm{O}_{5} / \mathrm{SiO}_{2}$ & 0.04 & 5 & 62 \\
3 & $\mathrm{P}_{2} \mathrm{O}_{5} / \mathrm{SiO}_{2}$ & 0.08 & 2 & 90 \\
4 & $\mathrm{P}_{2} \mathrm{O}_{5} / \mathrm{SiO}_{2}$ & 0.12 & 1.5 & 97 \\
5 & $\mathrm{P}_{2} \mathrm{O}_{5} / \mathrm{SiO}_{2}$ & 0.15 & 1.5 & 96 \\
6 & $\mathrm{Silphos}_{7}$ & 0.4 & 5 & 74 \\
7 & $\mathrm{Silphos}$ & 0.8 & 2.5 & 83 \\
8 & $\mathrm{Silphos}$ & 1.0 & 2 & 96 \\
9 & $\mathrm{Silphos}_{1}$ & 1.2 & 2 & 96 \\
10 & $\mathrm{P}_{2} \mathrm{O}_{5}$ & 0.04 & 2 & 74 \\
$11^{\mathrm{b}}$ & $\mathrm{PCl}_{3}$ & 0.05 & 3 & 52 \\
\hline
\end{tabular}

${ }^{a}$ Isolated yield. ${ }^{b}$ This reaction was carried out at $75{ }^{\circ} \mathrm{C}$

In another study, the condensation of dimedone with benzaldehyde was checked at different temperatures in the presence of $\mathrm{P}_{2} \mathrm{O}_{5} / \mathrm{SiO}_{2}$ and silphos under solvent-free conditions (Table 2). As Table 2 indicates, higher yields and shorter reaction times were observed at $80^{\circ} \mathrm{C}$ using both reagents.

Table 2. The reaction of dimedone $(2 \mathrm{mmol})$ with benzaldehyde $(1 \mathrm{mmol})$ using $\mathrm{P}_{2} \mathrm{O}_{5} / \mathrm{SiO}_{2}$ $(0.12)$ or silphos $(1.0 \mathrm{~g})$ at different temperatures under solvent-free conditions

\begin{tabular}{ccccc}
\hline Entry & Catalyst & Temperature, ${ }^{\circ} \mathrm{C}$ & Time, $\mathrm{h}$ & Yield $^{\mathrm{a}}, \%$ \\
\hline 1 & $\mathrm{P}_{2} \mathrm{O}_{5} / \mathrm{SiO}_{2}$ & r.t. & 20 & Trace \\
2 & $\mathrm{P}_{2} \mathrm{O}_{5} / \mathrm{SiO}_{2}$ & 60 & 5 & 67 \\
3 & $\mathrm{P}_{2} \mathrm{O}_{5} / \mathrm{SiO}_{2}$ & 70 & 3 & 81 \\
4 & $\mathrm{P}_{2} \mathrm{O}_{5} / \mathrm{SiO}_{2}$ & 80 & 1.5 & 97 \\
5 & $\mathrm{P}_{2} \mathrm{O}_{5} / \mathrm{SiO}_{2}$ & 90 & 1.5 & 97 \\
6 & $\mathrm{Silphos}$ & r.t. & 20 & Trace \\
7 & Silphos & 60 & 6 & 58 \\
8 & Silphos & 70 & 4 & 82 \\
9 & Silphos & 80 & 2 & 96 \\
10 & Silphos & 90 & 2 & 96 \\
\hline \multicolumn{5}{c}{}
\end{tabular}

To compare the efficiency as well as capacity of the solvent-free conditions with respect to solution conditions, the reaction of dimedone $(2 \mathrm{mmol})$ with benzaldehyde $(1 \mathrm{mmol})$ was tested in the presence of silica-supported $\mathrm{P}_{2} \mathrm{O}_{5}(0.12)$ or silphos $(1.0 \mathrm{~g})$ in several solvents $(5 \mathrm{~mL})$, such as $\mathrm{MeCN}, \mathrm{CH}_{2} \mathrm{Cl}_{2}, \mathrm{CHCl}_{3}$, THF and $\mathrm{EtOH}$ under reflux conditions. In these conditions, the product was produced in low yields and in long reaction times.

After optimization of the reaction conditions, dimedone was reacted with different types of aldehydes (including aromatic aldehydes possessing electron-releasing substituents, electron-withdrawing substituents and halogens on their aromatic rings as well as cinnamaldehyde) in the presence of the silica-supported phosphorus-containing catalysts. The results are displayed in Table 3. As it is shown in Table 3, all reactions were completed within 1-4 $\mathrm{h}$ and the desired products were obtained in good to excellent yields (76-98\%). Thus, our method is highly efficient and general. 
Table 3. The Synthesis of 1,8-doxo-octahydro-xanthene derivatives from dimedone and aldehydes in the presence of $\mathrm{P}_{2} \mathrm{O}_{5} / \mathrm{SiO}_{2}$ or silphos under solvent-free conditions at $80{ }^{\circ} \mathrm{C}$

\begin{tabular}{|c|c|c|c|c|}
\hline Entry & $\mathrm{R}$ & $\begin{array}{c}\mathrm{P}_{2} \mathrm{O}_{5} / \mathrm{SiO}_{2} \\
\text { Time, } \mathrm{h} / \mathrm{Yield}^{\mathrm{a}} \\
\end{array}$ & $\begin{array}{c}\text { Silphos } \\
\text { Time, }^{\mathrm{h} / Y} \\
\end{array}$ & M.P., (lit)[Ref] \\
\hline 1 & $\mathrm{C}_{6} \mathrm{H}_{5}$ & $1.5 / 97$ & $2 / 96$ & $\begin{array}{c}199-201 \\
(201-202)[12]\end{array}$ \\
\hline 2 & $p-\mathrm{ClC}_{6} \mathrm{H}_{4}$ & $1.15 / 97$ & $1.5 / 97$ & $\begin{array}{c}231-233 \\
(230-232)[12]\end{array}$ \\
\hline 3 & $m-\mathrm{ClC}_{6} \mathrm{H}_{4}$ & $2 / 87$ & $1.5 / 91$ & $\begin{array}{c}184-186 \\
(183-185)[11]\end{array}$ \\
\hline 4 & $o-\mathrm{ClC}_{6} \mathrm{H}_{4}$ & $2 / 95$ & $2 / 92$ & $\begin{array}{c}223-225 \\
(226-227)[11]\end{array}$ \\
\hline 5 & $p-\mathrm{NO}_{2} \mathrm{C}_{6} \mathrm{H}_{4}$ & $1 / 94$ & $1.5 / 97$ & $\begin{array}{c}223-225 \\
(222-224)[11]\end{array}$ \\
\hline 6 & $m-\mathrm{NO}_{2} \mathrm{C}_{6} \mathrm{H}_{4}$ & $1.5 / 97$ & $1 / 95$ & $\begin{array}{c}163-165 \\
(165-166)[11]\end{array}$ \\
\hline 7 & $o-\mathrm{NO}_{2} \mathrm{C}_{6} \mathrm{H}_{4}$ & $1.5 / 97$ & $2.5 / 90$ & $\begin{array}{c}249-251 \\
(252-254)[18]\end{array}$ \\
\hline 8 & $p-\mathrm{Br} \mathrm{C}_{6} \mathrm{H}_{4}$ & $1.5 / 93$ & $1.5 / 94$ & $\begin{array}{c}241-243 \\
(240-242)[12]\end{array}$ \\
\hline 9 & $o-\mathrm{BrC}_{6} \mathrm{H}_{4}$ & $2 / 87$ & $1.5 / 85$ & $\begin{array}{c}221-223 \\
(226-229)[11]\end{array}$ \\
\hline 10 & $p-\mathrm{CNC}_{6} \mathrm{H}_{4}$ & $1.5 / 88$ & $2 / 84$ & $\begin{array}{c}220-222 \\
(217-218)[11]\end{array}$ \\
\hline 11 & $p-\mathrm{CH}_{3} \mathrm{C}_{6} \mathrm{H}_{4}$ & $1 / 98$ & $1.5 / 95$ & $\begin{array}{c}213-215 \\
(215-216)[11]\end{array}$ \\
\hline 12 & $m-\mathrm{CH}_{3} \mathrm{C}_{6} \mathrm{H}_{4}$ & $1 / 96$ & $1.5 / 95$ & $\begin{array}{c}207-209 \\
(208-210)[18]\end{array}$ \\
\hline 13 & $o-\mathrm{CH}_{3} \mathrm{C}_{6} \mathrm{H}_{4}$ & $3 / 80$ & $2.5 / 85$ & $\begin{array}{c}210-212 \\
(210-212)[18]\end{array}$ \\
\hline 14 & $p-\mathrm{CH}_{3} \mathrm{OC}_{6} \mathrm{H}_{4}$ & $2 / 93$ & $2 / 91$ & $\begin{array}{c}241-243 \\
(242-243)[11]\end{array}$ \\
\hline 15 & $m-\mathrm{CH}_{3} \mathrm{OC}_{6} \mathrm{H}_{4}$ & $3 / 86$ & $2.5 / 85$ & $\begin{array}{c}161-163 \\
(161-162)[11]\end{array}$ \\
\hline 16 & $o-\mathrm{OHC}_{6} \mathrm{H}_{4}$ & $1 / 95$ & $1.15 / 93$ & $\begin{array}{c}198-200 \\
(194-196)[18]\end{array}$ \\
\hline 17 & $\begin{array}{c}3,4- \\
\left(\mathrm{CH}_{3} \mathrm{O}\right)_{2} \mathrm{C}_{6} \mathrm{H}_{3}\end{array}$ & $3 / 87$ & $2 / 90$ & $\begin{array}{c}178-180 \\
(175-176)[11]\end{array}$ \\
\hline 18 & $\begin{array}{c}3,4,5- \\
\left(\mathrm{CH}_{3} \mathrm{O}\right)_{3} \mathrm{C}_{6} \mathrm{H}_{2} \\
5-\mathrm{Br}-2\end{array}$ & $4 / 76$ & $3.5 / 83$ & $\begin{array}{c}201-203 \\
(205-208)[12]\end{array}$ \\
\hline 19 & $\begin{array}{l}5-\mathrm{Br}-2- \\
\mathrm{OHC}_{6} \mathrm{H}_{3}\end{array}$ & $1 / 97$ & $1.5 / 97$ & $\begin{array}{c}250-252 \\
(249-252)[18]\end{array}$ \\
\hline 20 & $\mathrm{C}_{6} \mathrm{H}_{5} \mathrm{CH}=\mathrm{CH}$ & $2.5 / 89$ & $1.5 / 94$ & $\begin{array}{c}172-174 \\
(174-176)[18]\end{array}$ \\
\hline 21 & $p-\mathrm{OHCC}_{6} \mathrm{H}_{4}$ & $4 / 95$ & $3.5 / 92$ & $\begin{array}{c}>300 \\
(>300)[18]\end{array}$ \\
\hline
\end{tabular}




\section{Conclusion}

In conclusion, we have developed a new method for the synthesis of 1,8-dioxooctahydroxanthenes using two heterogeneous catalysts, $\mathrm{P}_{2} \mathrm{O}_{5} / \mathrm{SiO}_{2}$ and silphos. The simple experimental and work-up procedures, efficiency, high yields, relatively short reaction times, generality, application of inexpensive catalysts and compliance with the green chemistry protocols are the advantages of the present method.

\section{Acknowledgement}

The authors thank the Research Committee of Persian Gulf University for the financial support of this work.

\section{References}

1. Clark J H, Acc Chem Res., 2002, 35, 791-797.

2. Salehi P, Zolfigol M A, Shirini F and Baghbanzadeh M, Curr Org Chem., 2006, 10, 2171-2189 (Review).

3. a) Mohammadizadeh M.R, Hasaninejad A, Bahramzadeh $M$ and Khanjarlou Z S, Synth Commun., 2009, 39, 1152-1162; b) Hasaninejad A, Niknam K, Zare A, Farsimadan E and Shekouhy M, Phosphorus, Sulfur Silicon Relat Elem., 2009, 184, 147; c) Hasaninejad A, Zare A, Sharghi H and Shekouhy M, ARKIVOC, 2008, xi, 64; d) Hasaninejad A, Zare A, Balooty L and Mehregan H, Synth Commun., 2010, 40, 3488.

4. a) Iranpoor N, Firouzabadi H, Jamalian A and Kazemi F, Tetrahedron, 2005, 61, 5699;

b) Iranpoor N, Firouzabadi H and Jamalian A., Tetrahedron Lett., 2005, 46, 7963-7966;

c) Iranpoor N, Firouzabadi H and Jamalian A, Synlett., 2005, 9, 1447-1449; d) Iranpoor N, Firouzabadi H and Jamalian A, Phosphorus, Sulfur Silicon Relat Elem, 2006, 181, 2615.

5. a) Menchen S M, Benson S C, Lam J Y L, Zhen W, Sun D, Rosenblum B B, Khan S H and Taing M, U. S. Patent, 2003, 6,583,168, Chem Abstr., 2003, 139, p54287f; b) Hunter R C and Beveridge T J, Appl Environ Microibiol., 2005, 71, 2501; c) Ahmad M, King T A, Ko D-K, Cha B H and Lee J, J Phys D Appl Phys., 2002, 35, 1473.

6. Lambert R W, Martin J A, Merrett J H, Parkes K E B and Thomas G J, PCT Int Appl. WO 1997, 9706178; Chem Abstr., 1997, 126, p212377y.

7. Hideo T, Jpn Tokkyo Koho, 1981, 56,005,480; Chem Abstr., 1981, 95, 80922b.

8. Llama E F, Campo C D, Capo M and Anadon M, Eur J Med Chem., 1989, 24, 391.

9. Ion R M, Frackowiak D, Planner A and Wiktorowicz K, Acta Biochim Pol., 1998, 45, 833.

10. Poupelin J P, Saint-Rut G, Foussard-Blanpin O, Narcisse G, Uchida-Ernouf G and Lacroix R, Eur J Med Chem., 1978, 13, 67-71.

11. Zhang Z-H and Liu Y-H, Catal Commun.. 2008, 9, 1715-1719.

12. Kantevari S, Bantu R and Nagarapu L J, Mol Catal A: Chem., 2007, 269, 53-57.

13. Das B, Thirupathi P, Reddy K R, Ravikanth B and Nagarapu L, Catal Commun., 2007, 8, 535-538.

14. Shaterian H.R, Hosseinian A and Ghashang M, Phosphorus, Sulfur and Silicon, 2008, 183, 3136.

15. Seyyedhamzeh M, Mirzaei P and Bazgir A, Dyes Pigments, 2008, 76, 836.

16. ShakibaeiGI, Mirzaei P and Bazgir A, Appl Catal A: Gen., 2007, 325, 188-192.

17. Das B, Thirupathi P, Mahender I, Reddy V S and Rao Y K, J Mol Catal A: Chem., 2006, 247, 233-239.

18. Dabiri M, Baghbanzadeh M and Arzroomchilar E, Catal Commun., 2008, 9, 939-942.

19. Kantevari S, Bantu R and Nagarapu L, ARKIVOC, 2006, xvi, 136-148.

20. a) Hasaninejad A, Zare A and Shekouhy M, Ameri-Rad J, Green Chem., 2011, 13, 958-964; b) Hasaninejad A, Zare A and Shekouhy M, Tetrahedron, 2011, 67, 390; c) Hasaninejad A, Zare A, Shekouhy M and Ameri Rad J, J Comb Chem., 2010, 12, 844-849. 